\title{
HOIDS-Based Detection Method of Vicious Event in Large Networks
}

\author{
Dong Hwi Lee ${ }^{1}$, Jeom Goo Kim ${ }^{2}$, and Kuinam J. Kim ${ }^{1}$ \\ ${ }^{1}$ Dept. of Information Security Kyonggi Univ., Korea \\ harap123 adaum. net \\ ${ }^{2}$ Dept. of Computer Science Namseoul Univ., Korea
}

\begin{abstract}
It is very crucial in the field of security control to acquire the capability of promptly coping with various threatening elements in cyber world such as vicious worms, virus and hackings that cause enormous damage and loss across the nation within a rather short term period like the large scale network paralyzed by vicious traffic, disturbance of electronic commerce, etc. As such, it can be the fundamental measure on these sorts of threats to establish the new method of detecting the similar threats as well as to reinforce the user's recognition of security. The purpose of this study is to analyze the problems in the existing IDS and TMS, which are monolithic in terms of detection method, and further to suggest the improved detection method and HOIDS system which is recently introduced and in test operation.
\end{abstract}

\section{Introduction}

The large network has been entering into the era of the ubiquitous environment, creating the extravagant economic value. Nevertheless, the increase of cyber terrors such as computer virus and vicious hackings threats the foundation of the information society. Accordingly, there is high demand on the solution of these threats. Above all, there is a strong agreement on the importance of security control on the vicious worms and virus, which cause enormous damage and loss across the nation within a rather short term period like the large network paralyzed by vicious traffic, disturbance of electronic commerce, etc.

While the biological virus and worms generate spontaneously, the computer virus and worms are deliberately improved by the creators. This leads to the difficulty in detecting the threats on the early stage, and shows the limitation of the existing studies. As a result, the early alert system for the cyber terrors is not in real use. The importance of early detection method is gaining the attention to overcome such limitations. Furthermore, the new virus becomes more sophisticated, automatic and diverse. The lack of human resource and technology, and the individual operation of information security system in the security institutes make the security control more difficult.

In this paper we design the Honeynet based early alert system to reinforce the capability of coping with the cyber attack in the medium/large scale network, and the 
efficient detection method in each stage to improve the existing detection methods. First, we operated the HOIDS (i.e., Honey Net based IDS for early detection) that enables to forecast and judge the threat beforehand. At the same time, we implemented the security measures such as analysis of correlation of various events and threats blocking by loading the data of events collected by IDS into the consolidated Database.

\section{Related Work}

Honey Systems are decoy servers or systems setup to gather information regarding an attacker or intruder into your system. That is, Honey Systems mean the systems revealing their effectiveness under the hacker's attacks, and collecting the information on the intruder and then based upon such information providing the information which helps to reinforce the security. As guessing its name meaning a honey pot, the Honeypot System is used to decoy someone. [1,2] Most studies on Honeynet and Honeypot are utilized to identify the behavioral patterns of vicious virus and worms and handle them. Especially, the Honeynet system is effective in detecting the vicious traffic in the large scale network [3].

Hellerstein estimated the volume of weekly traffic in the wired-network by applying "the Seasonal ARIMA model" for early alert on traffic[4]. In addition, F.Zang forecasted the traffic of wireless communication with the Seasonal ARIMA model[5], Groschwitz suggested the method of easily identifying both short-term and long-term errors in the traffic with each filtered signal by analyzing the original traffic in time cycle based upon the "Wavaelet Analysis[6]." Y. Shu suggest the appropriate method of forecasting the non-lineal and non-rectifying traffic in the high speed network with the "Fuzzy-AR model [7]." The most common ground of Hellerstein, F.Zang and Y.Shu is the fact that they all suggest the model enabling to forecast the possible situations occurring in the enormous volume of traffic and errors based upon the critical-value based analysis. However, the attribute of recent threats causing the traffic congestion Is evolving into the artificial intelligence method and diffusive-attacking method with irregular speed from the simple port attack or escalating attack. [8] In this circumstance, it is difficult to forecast the new cyber threat relying on the analytic method based on the quantitative measurement of traffic. This study invents the new method blocking the threats beforehand by acquiring the necessary information on the early stage instead of the quantitative measurement.

Most vicious worms terrifying the internet users examine their performance in numerous hackings and then spread widely over the incubation period of weeks till moths. Accordingly, we need to understand that such vicious worms can be prevented by early identifying them with the relevant information.

The study of correlation of threat and weakness in the network make it possible to forecast the cyber threat through the analysis of the correlation of N-IDS and VAS[9], Considering the recent patterns of cyber threat and weakness of N-IDS(Network Intrusion Detection System), there is a limitation in he forecast and alert on the cyber threat based upon the analytic model suggested in the above study. 


\section{Design of HOIDS Based Detection Method}

\subsection{Diagram of Structure Applying HOIDS}

The structure of Honeynet System consists of Honeynet System and Honeypot System. Here the structure is simplified with Honeynet System for early detection. First, IP in the specific section among the whole system is transmitted to Honeynet and specific IP is detected. The malign traffic would be primarily detected, which differs from the existing intrusion detection system. This considers each clue regarding the new threat meaningfully. Honeypot System adopts the result of each clue and input the pattern of detection in individual security equipments.

The structure with 3 stages, firewall section, server section and client section from the exit point, has additional gateway section for web traffic and inter-network traffic filtering and internal network gateway section. As illustrated in the below Fig. 1, the structure is composed of security level with 4 stages. The general level of firewall is unable to get the expected blocking effect since the rapid increase of normal and abnormal traffic leads to the enormous increase of inflowing virus. Therefore, the additional gateway section is required to prevent the vicious traffic from entering into the readjusted network. The inter-network traffic filtering can detect the unknown and undetected vicious traffic. As a detection method, the HOIDS sensor is installed in the initial inflow section and each gateway section. Each IPS in the gateway blocks the internal traffic of the intruded vicious events, which serves as a firewall and detection section.

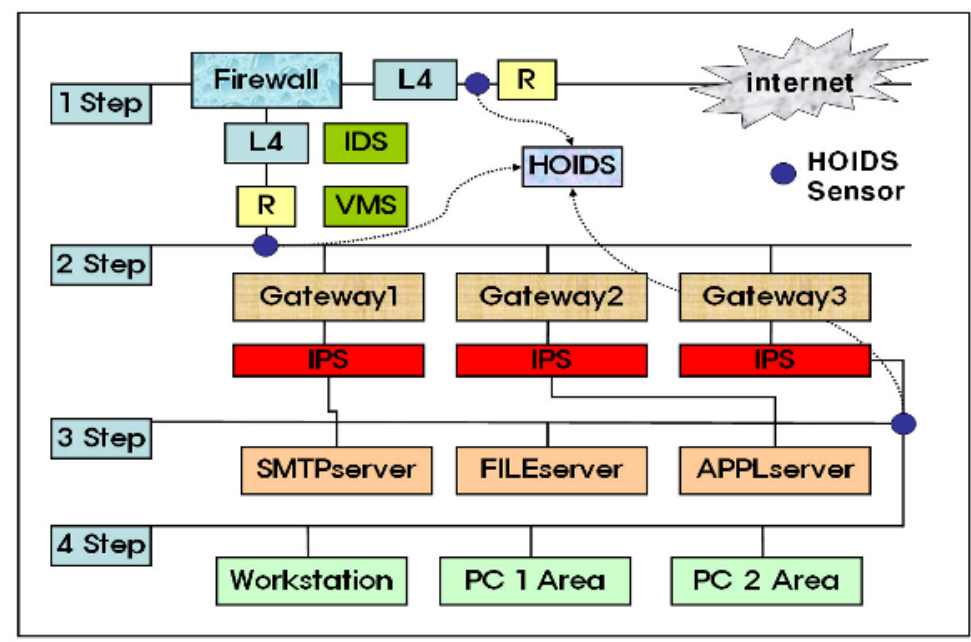

Fig. 1. The Diagram of Improved Structure applying HOIDS

\subsection{Design of Security Structure Applying HOIDS}

HOIDS is the most important module in this improved security structure. The composition and operating structure of HOIDS are illustrated in the below Fig. 2. The cyber 


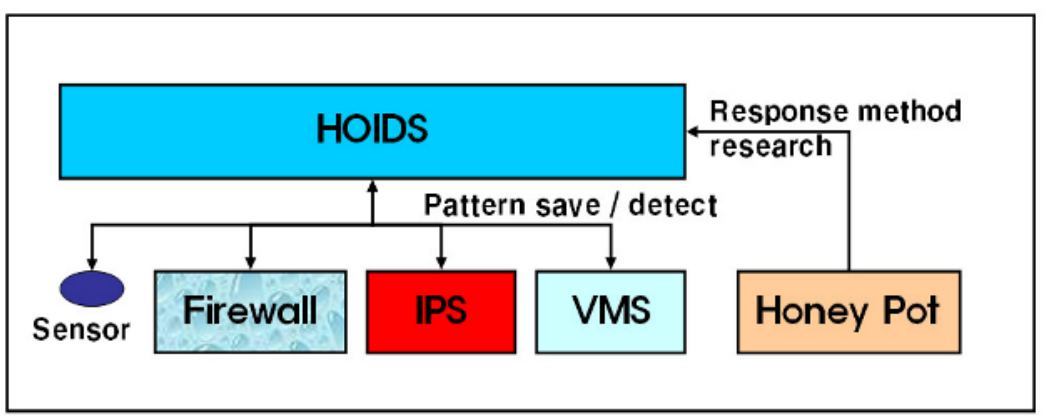

Fig. 2. HOIDS operating structure

threat elements from the event in the individual unused IP sections are collected in the wide section of network. The potential weakness is determined through the analysis of the collected information against the pattern of the events in each security equipment. The detection method of each security equipment will be improved by analyzing the way of expansion and increase of vicious events in the Honyepot.

\subsection{The Detection Process}

The detection process in HOIDS is illustrated in the Fig. 3 and 4. Firstly, the large scale network is divided into individual unused IP detection sections, collecting the information with each sensor by gateway. Secondly, the vicious events are divided into the matching events and non-matching events and then its threatening elements are analyzed and evaluated as shown in the Fig. 3. The detection of non-matching events are performed to detect the varied vicious events on an early stage.

Thirdly, the events and information collected in various security equipments and network are fed into HOIDS Database. Fourthly, the system consolidates and evaluates the level of weakness of network and properties, the level of current expansion and distribution of worm virus, the scope of each virus variety, and the internal and external information on the threat.

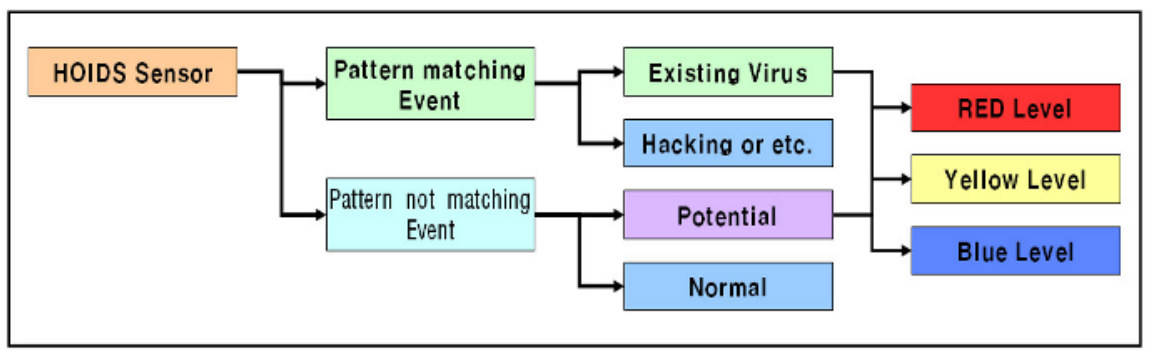

Fig. 3. The HOIDS pattern matching method 


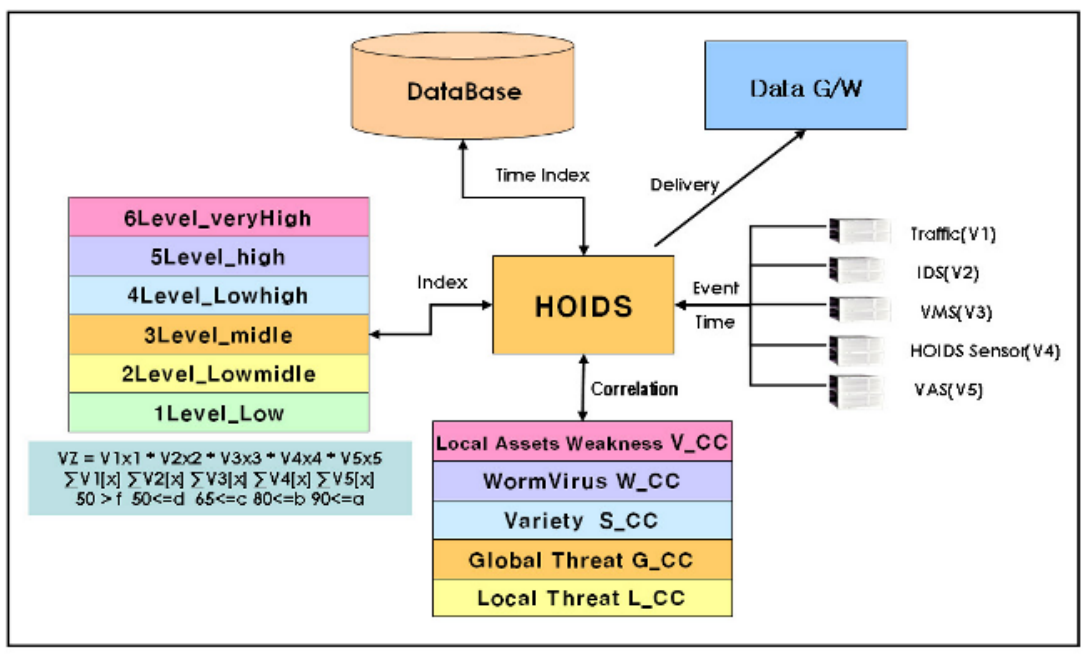

Fig. 4. The Evaluation Method by HOIDS Events

Fifthly, it evaluates the threat by stages. Categorizes them by the level of threat and transmits such information into each security equipment for blocking. Sixthly, each evaluation results are fed into the Database and standardized by time frame. The standardized information is accumulated and defined as evaluation value for the accuracy of the consolidated evaluation.

\section{Performance Analysis}

\subsection{Analysis Environment}

Two large scale networks at the speed of more than $1 \mathrm{G}$ byte test with the same security equipment for one month. The examination was performed toward real works in the network of $\mathrm{K}$ institute for the common analysis environment. Now that the networks were not installed with the security equipment having the improved infrastructure, the preparation work was required to install the measuring equipments and set up the appropriate environment for the targeted examination. The said K institute has 500 servers in various sizes for intranet system, 10,000 PCs for the client's workstation and 20,000 internal users.

The network structure consists of intranets and dual line network linked at the speed of $500 \mathrm{Mbps}$ with the external networks. The intrusion blocking system is installed at the entrance of the intranet. In the internal structure of the intranet, the PC is connected with the server having separate mail searching system. PCs are installed with individual virus vaccine.

In terms of the size of traffic, it has a large volume handling around 500 million packets per day and regularly around 50,000 sessions of network. The security equipment consists of 4 Giga bit Firewalls (including backup lines), 8 IDSs (Sun V880), 4 Giga bit IPSs, 2 VMS Servers and a single ESM (Sun ENT3500). 
The A network is installed with the HOIDS and improved security structure as shown in the Fig. 5.

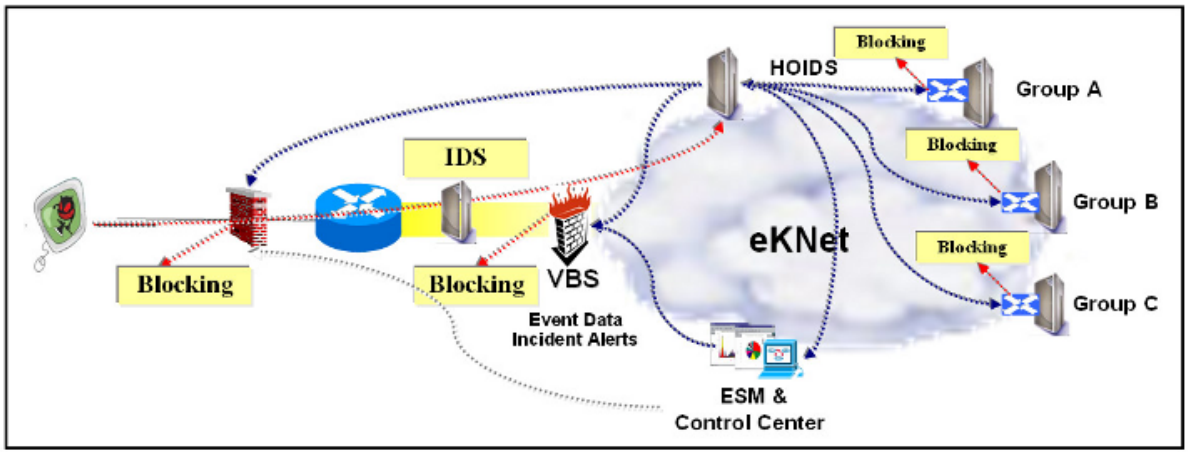

Fig. 5. A Network's analysis model structure

The B network is installed with the existing security structure as shown in the Fig. 6.

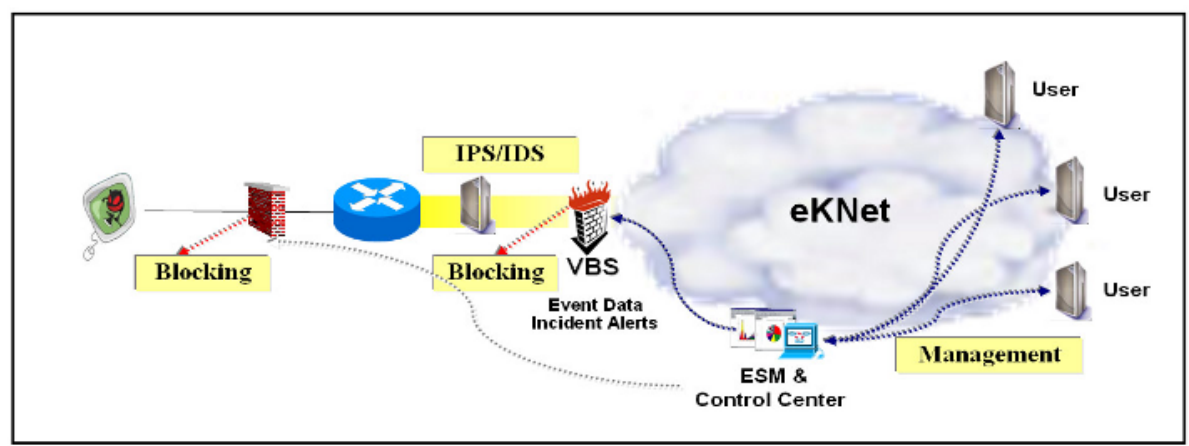

Fig. 6. B Network's analysis model structure

In the A network, the information about HOIDS cyber threat is announced through the company network and the application of security equipment rules with analysis result of HOIDS. The same security equipments such as VMS, F/W, IDS and IPS are installed.

We evaluated the performance by examining the VMS statistics for 3 months. For two months, the statistics were calculated without the application of the improved security structure in both A network and B network. For the third month, they were calculated by applying the improved security structure in the A network.

\subsection{Analysis Result}

The results in the Table 1 and Table 2 show the similar developments in the average for the first and second month. On the other hand, the A network with the improved security structure and HOIDS has more than $40 \%$ decreases in the number of worm virus comparing with one of B network for the third month. In the graphs of the Fig. 7 
Table 1. The number of Virus Infection in the A network

\begin{tabular}{|c|c|c|c|}
\hline Month & $\mathbf{1}$ & $\mathbf{2}$ & $\mathbf{3}$ \\
\hline Worm & 143,221 & 112,684 & 58,129 \\
\hline Trojan & 21,682 & 15,703 & 9,823 \\
\hline Mail & 39,458 & 38,990 & 18,536 \\
\hline Total & $\mathbf{2 0 4 , 3 6 8}$ & $\mathbf{1 6 7 , 3 8 5}$ & $\mathbf{8 6 , 4 9 7}$ \\
\hline Vs. pre month & $100 \%$ & $82 \%$ & $52 \%$ \\
\hline
\end{tabular}

Table 2. The number of Virus Infection in the B network

\begin{tabular}{|c|c|c|c|}
\hline Month & $\mathbf{1}$ & $\mathbf{2}$ & 3 \\
\hline Worm & 138,933 & 156,129 & 121,785 \\
\hline Trojan & 19,872 & 12,763 & 13,165 \\
\hline Mail & 51,240 & 33,150 & 28,775 \\
\hline Total & $\mathbf{2 1 0 , 0 5 2}$ & $\mathbf{2 0 2 , 0 5 0}$ & $\mathbf{1 6 3 , 7 3 4}$ \\
\hline Vs. pre month & $100 \%$ & $96 \%$ & $81 \%$ \\
\hline
\end{tabular}

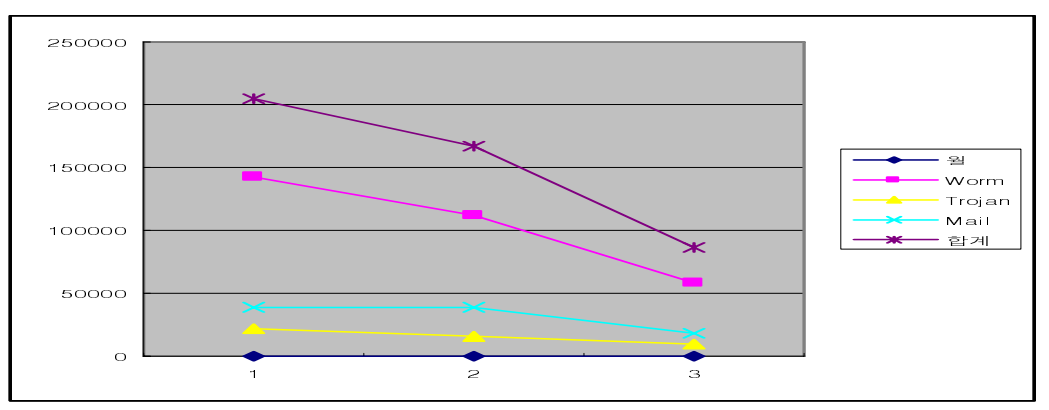

Fig. 7. The graph of Virus in A Network

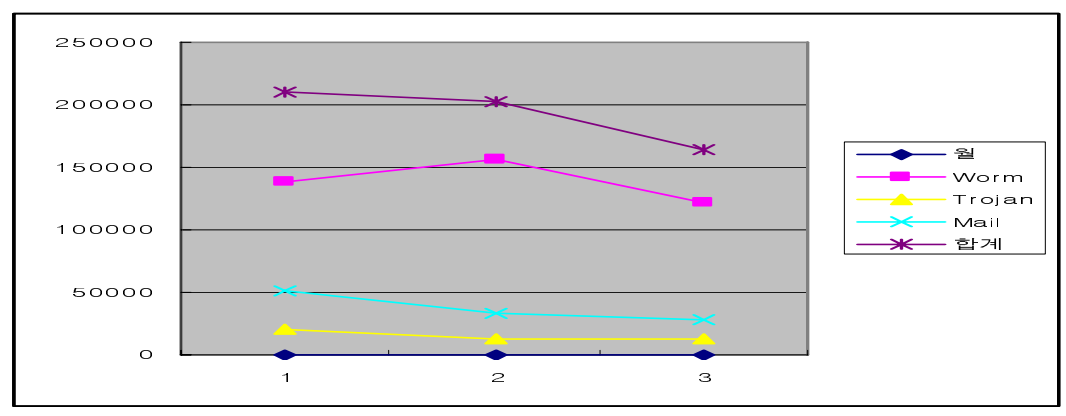

Fig. 8. The graph of Virus in B Network 
and Fig. 8, the A network has more than 50\% decrease and the B network has around $20 \%$ decrease comparing with the second month. In other words, we can estimate that the A network have further decrease of around $30 \%$. Especially we can note that this is effective in blocking the worms causing vicious traffic in the certain pattern and the worms spreading out through the mails.

\section{Conclusion}

This paper focused on the improved and efficient model of the large scale network structure and detection method in terms of the structure to cope with the new vicious traffic patterns gaining their strength of threat. For this purpose, the security equipments with the improved structure were installed by the level of threat and service. HOIDS was installed as a detection method to early detect the vicious events, which were not detected in the existing method, and prevent them from spreading out with a rapid blocking. We conclude that the vicious traffic can be efficiently blocked by the consolidated approach such as early detection and recognition of the exact pattern of vicious traffic, and blocking and prevention of such traffic.

\section{Acknowledgement}

This research was supported by the MIC (Ministry of Information and Communication), Korea, under the ITRC (Information Technology Research Center) support program supervised by the IITA (Institute of Information Technology Assessment).

\section{References}

1. Nicolas Vanderavero, Xavier Brouckaert, Olivier Bonaventure, Baudouin Le Charlier. "The HoneyTank: a Scalable Approach to Collect Malicious Internet traffic".IEEE2004 RTSS04, Session 1, 2004.12

2. Dong-il Seo, Yang-seo Choi, Sang-Ho Lee. "Design and Development of Real Time Honeypot System for Collecting the Information of Hacker Activity", KIPS 2004, VOL. 10 NO.01 pp.1941 1944, 2003.05

3. http://www.Honeynet.org,"Honeynet Project Overview", 2005.4

4. J.L Hellerstein, F.Zhang, P. Shahabuddin. "A Statistical Approach to Predictive Detection", Computer Networks, vol 35, pp77-95, 2001

5. F.Zang, J.L Hellerstein. “An Approach to On-line Predictive Detection”, In Preoceedings Of 8th International Symposium on Modeling. ASCTS, 2000

6. N.K Groschwitz and G. C. Polyzos. "A Time Series Model of Long-Term NAFNET Backbone Traffic", In proceedings of IEEE International Conference on Communications, 1994

7. Y. Shu, M. Yu, J Liu. "Wireless traffic modeling and prediction using seasonal ARIMA models", In proceedings of IEEE International Conference on Communications,v.3, 2003

8. http://info.ahnlab.com/ahnlab/report_view.jsp?num=416

9. Ho-Kun Moon, Jin-gi Choe, Yu Kang, Myung-soo Rhee. "The study of correlation of threat and weakness in the network make it possible to forecast the cyber threat through the analysis of the correlation of N-IDS and VAS", Korea Institute of Information Security \& Cryptology, 2005 